\title{
Rheumatologic manifestations of primary immunodeficiency diseases: comment on the study by Dimitriades et al.
}

\author{
Bahtiyar Toz ${ }^{1} \cdot$ Burak Erer $^{1}$
}

Received: 25 April 2016 / Accepted: 25 April 2016/Published online: 3 May 2016

(C) International League of Associations for Rheumatology (ILAR) 2016

\section{Dear Editor,}

We read with interest the review entitled "Rheumatologic manifestations of primary immunodeficiency diseases" by Dimitriades et al. [1]. This is a well-written review providing guidance for rheumatologists regarding when they should consider primary immunodeficiency diseases and also explores how these immunodeficiency states may be underlying their rheumatologic disorder. We would like to comment on this review regarding other associations between rheumatologic disorders and primary immunodeficiency diseases.

In addition to inflammasome-related disorders, interferonopathies such as chronic atypical neutrophilic dermatosis with lipodystrophy and elevated temperature and STINGassociated vasculopathy with onset in infancy may be seen in the setting of autoinflammatory disorders. Furthermore, PLC $\gamma 2$ associated antibody deficiency and immune dysregulation, autoinflammation and PLC $\gamma 2$-associated antibody deficiency and immune dysregulation, HOIL-1, and NF-kB disorders including Blau syndrome and early onset enterocolitis, familial psoriasis may be seen in association with autoinflammatory disorders [2].

Bahtiyar Toz

btoz@istanbul.edu.tr

Division of Rheumatology, Department of Internal Medicine, Istanbul Faculty of Medicine, Istanbul University, Istanbul, Turkey
Secondly, adenosine deaminase 2 protein (encoded by CECR1 gene) deficiency has recently been defined and may be associated with recurrent fever and early onset vasculopathy mimicking classical polyarteritis nodosa characterized by stroke and microaneurysms, inflammation, and mild immunodeficiency [3]. Thus, this entity may also be included in the list.

Lastly, hyper-immunoglobulin E syndrome is a rare primary immune deficiency disease characterized by skin and lung infections and dermatitis. Vasculitis leading to stenosis, occlusion, and aneurysm formation has been reported in this syndrome [4].

We suggest the inclusion of these entities in this extensive review which provides guidance in the setting of rheumatologic presentations in association with primary immunodeficiency diseases.

Compliance with ethical standards

Disclosures None.

\section{References}

1. Dimitriades VR, Sorensen R (2016) Rheumatologic manifestations of primary immunodeficiency diseases. Clin Rheumatol 35(4):843-50

2. Giannelou A, Zhou Q, Kastner DL (2014) When less is more: primary immunodeficiency with an autoinflammatory kick. Curr Opin Allergy Clin Immunol 14(6):491-500

3. De Jesus AA, Canna SW, Liu Y et al (2015) Molecular mechanisms in genetically defined autoinflammatory diseases: disorders of amplified danger signaling. Annu Rev Immunol 33:823-74

4. Yavuz H, Chee R (2010) A review on the vascular features of the hyperimmunoglobulin E syndrome. Clin Exp Immunol 159(3):238-44 